\title{
REAL THETA CHARACTERISTICS AND AUTOMORPHISMS OF A REAL CURVE
}

\author{
INDRANIL BISWAS ${ }^{\bowtie}$ and SIDDHARTHA GADGIL
}

(Received 21 April 2009; accepted 11 July 2009)

Communicated by F. Calegari

\begin{abstract}
Let $X$ be a geometrically irreducible smooth projective curve defined over $\mathbb{R}$, of genus at least 2 , that admits a nontrivial automorphism, $\sigma$. Assume that $X$ does not have any real points. Let $\tau$ be the antiholomorphic involution of the complexification $x_{\mathbb{C}}$ of $X$. We show that if the action of $\sigma$ on the set $\mathcal{S}(X)$ of all real theta characteristics of $X$ is trivial, then the order of $\sigma$ is even, say $2 k$, and the automorphism $\tau \circ \widehat{\sigma}^{k}$ of $X_{\mathbb{C}}$ has a fixed point, where $\widehat{\sigma}^{k}$ is the automorphism of $X \times \mathbb{R} \mathbb{C}$ defined by $\sigma$. We then show that there exists $X$ with a real point and admitting a nontrivial automorphism $\sigma$, such that the action of $\sigma$ on $\mathcal{S}(X)$ is trivial, while $X /\langle\sigma\rangle \neq \mathbb{P}_{\mathbb{R}}^{1}$. We also give an example of $X$ with no real points and admitting a nontrivial automorphism $\sigma$, such that the automorphism $\tau \circ \widehat{\sigma}^{k}$ has a fixed point, the action of $\sigma$ on $\mathcal{S}(X)$ is trivial, and $X /\langle\sigma\rangle \neq \mathbb{P}_{\mathbb{R}}^{1}$.
\end{abstract}

2000 Mathematics subject classification: primary 14P25; secondary 14F10.

Keywords and phrases: real curve, real theta characteristic, automorphism.

\section{Introduction}

Let $X$ be a smooth complex projective curve of genus $g$, with $g \geq 2$. Assume that $X$ admits nontrivial automorphisms; fix a nontrivial automorphism $\sigma$ of $X$. In [3] it was shown that if $\sigma$ fixes all the theta characteristics of $X$ pointwise, then $X$ is hyperelliptic and $\sigma$ is the unique hyperelliptic involution of $X$ (this was also proved in [5]). Our aim here is to address a similar question for curves defined over the field of real numbers.

Let $X$ be a geometrically irreducible smooth projective curve defined over $\mathbb{R}$. Let

$$
\tau: X \times_{\mathbb{R}} \mathbb{C} \longrightarrow X \times_{\mathbb{R}} \mathbb{C}
$$

be the antiholomorphic involution of the Riemann surface $X \times \mathbb{R}$. The genus of $X$, which will be denoted by $g$, is assumed to be at least 2 . Let $\operatorname{Pic}^{g-1}(X)_{\mathbb{R}}$ denote the real points of the Picard variety $\mathrm{Pic}^{g-1}(X)$. The real theta characteristics of $X$ are all those points of $\mathrm{Pic}^{g-1}(X)_{\mathbb{R}}$ which are square roots of the real point of $\operatorname{Pic}^{2 g-2}(X)$ given by

(C) 2010 Australian Mathematical Publishing Association Inc. 1446-7887/2010 \$16.00 
the cotangent line bundle $K_{X}$ of $X$. Therefore any automorphism of $X$ acts on the set of real theta characteristics of $X$. The set of real theta characteristics of $X$ will be denoted by $\mathcal{S}(X)$. It should be mentioned that in general not all points of $\mathcal{S}(X)$ represent some real algebraic line bundle over $X$.

Our first result is the following (see Theorem 2.3).

THEOREM 1.1. Let $X$ be a geometrically irreducible smooth projective curve, of genus at least 2 , defined over the field of real numbers, without any real points. Assume that $X$ admits a nontrivial automorphism $\sigma$. Then at least one of the following is true.

(1) The action of $\sigma$ on $\mathcal{S}(X)$ is nontrivial.

(2) The order of $\sigma$ is even, say $2 k$, and the involution $\tau \circ \widehat{\sigma}^{k}$ of $X_{\mathbb{C}}$ has a fixed point, where $\widehat{\sigma}^{k}$ is the automorphism of $X_{\mathbb{C}}$ defined by $\sigma$.

In Section 3 we show that there exists $X$ with a real point and admitting a nontrivial automorphism $\sigma$, such that:

(1) the action of $\sigma$ on $\mathcal{S}(X)$ is trivial; and

(2) $X /\langle\sigma\rangle \neq \mathbb{P}_{\mathbb{R}}^{1}$.

We also give an example of $X$ with no real points and admitting a nontrivial automorphism $\sigma$, such that:

(1) the automorphism $\tau \circ \widehat{\sigma}^{k}$ has a fixed point;

(2) the action of $\sigma$ on $\mathcal{S}(X)$ is trivial; and

(3) $X /\langle\sigma\rangle \neq \mathbb{P}_{\mathbb{R}}^{1}$.

We also complete the case of odd theta characteristics which was left out in [3]. More precisely, we prove the following (see Proposition 4.2).

PROPOSITION 1.2. Let $Y$ be a connected smooth complex projective curve of genus $g$, with $g \geq 2$, that admits a nontrivial automorphism $\sigma$. If $\sigma$ fixes all the odd theta characteristics of $Y$ pointwise, then $Y$ is hyperelliptic and $\sigma$ is the unique hyperelliptic involution of $Y$.

It was noted in [3] that the corresponding statement for even theta characteristics on $X$ is valid; see [3, p. 496, Proposition 2.3].

\section{Real theta characteristics}

Let $X$ be a geometrically irreducible smooth projective curve defined over the field of real numbers. Let

$$
X_{\mathbb{C}}:=X \times \mathbb{R} \mathbb{C}
$$

be the complexification of $X$. Therefore $X_{\mathbb{C}}$ is an irreducible smooth projective curve defined over $\mathbb{C}$. In other words, $X_{\mathbb{C}}$ is a compact connected Riemann surface. The Galois group $\operatorname{Gal}(\mathbb{C} / \mathbb{R})=\mathbb{Z} / 2 \mathbb{Z}$ acts on $X_{\mathbb{C}}$ through an antiholomorphic involution

$$
\tau: X_{\mathbb{C}} \longrightarrow X_{\mathbb{C}}
$$

The real points of $X$ are precisely the fixed points of this involution $\tau$. 
Given a holomorphic line bundle $L$ on the Riemann surface $X_{\mathbb{C}}$, let $\bar{L}$ denote the $C^{\infty}$ complex line bundle on $X_{\mathbb{C}}$ whose underlying smooth real vector bundle of rank 2 is identified with the smooth real vector bundle of rank 2 underlying $L$, while for $x \in X_{\mathbb{C}}$, the complex structure of a fiber $\bar{L}_{x}$ is the conjugate of the complex structure of the fiber $L_{x}$. The pullback $\tau^{*} \bar{L}$ has a natural holomorphic structure, where $\tau$ is the antiholomorphic involution of $X_{\mathbb{C}}$ in (2.1). A smooth section of $\tau^{*} \bar{L}$ defined over an analytic open subset $U \subset X_{\mathbb{C}}$ is holomorphic if and only if the corresponding section of $L$ over $\tau(U)$ is holomorphic.

For any $d \in \mathbb{Z}$, let $\operatorname{Pic}^{d}(X)$ be the Picard variety of $X$ for degree $d$. The set of real points of $\operatorname{Pic}^{d}(X)$ will be denoted by $\operatorname{Pic}^{d}(X)_{\mathbb{R}}$. We note that $\operatorname{Pic}^{d}(X)_{\mathbb{R}}$ parameterizes all holomorphic line bundles $L$ of degree $d$ over $X_{\mathbb{C}}$ such that $\tau^{*} \bar{L}$ is holomorphically isomorphic to $L$, where $\tau$ is defined in (2.1).

The real algebraic line bundles over $X$ of degree $d$ form a subset of $\operatorname{Pic}^{d}(X)_{\mathbb{R}}$. However, in general not every point of $\operatorname{Pic}^{d}(X)_{\mathbb{R}}$ corresponds to some real algebraic line bundle over $X$ of degree $d$. To explain this, let $\xi$ be a real algebraic line bundle of degree $d$ over $X$. Then the base change $\xi_{\mathbb{C}}:=\xi \otimes_{\mathbb{R}} \mathbb{C}$ is a holomorphic line bundle over $X_{\mathbb{C}}$ of degree $d$. The Galois group $\operatorname{Gal}(\mathbb{C} / \mathbb{R})=\mathbb{Z} / 2 \mathbb{Z}$ acts on $\xi_{\mathbb{C}}$ through an isomorphism of holomorphic line bundles

$$
f: \xi \longrightarrow \tau^{* \bar{\xi}}
$$

such that the composition

$$
\xi \stackrel{f}{\longrightarrow} \tau^{*} \bar{\xi} \stackrel{\tau^{*} \bar{f}}{\longrightarrow} \tau^{*} \overline{\tau * \bar{\xi}}=\xi
$$

is the identity automorphism of $\xi$. Conversely, any pair $\left(\zeta, f_{0}\right)$, where $\zeta$ is a holomorphic line bundle over $X_{\mathbb{C}}$ of degree $d$ and

$$
f_{0}: \zeta \longrightarrow \tau^{*} \bar{\zeta}
$$

is a holomorphic isomorphism of line bundles such that the composition

$$
\zeta \stackrel{f_{0}}{\longrightarrow} \tau^{*} \bar{\zeta} \stackrel{\tau^{*} \overline{f_{0}}}{\longrightarrow} \tau^{*} \bar{\tau} \overline{\bar{\zeta}}=\zeta
$$

is the identity automorphism of $\zeta$, define a real algebraic line bundle over $X$ of degree $d$. The point to note is that in general there are examples of holomorphic line bundles $\zeta$ over $X_{\mathbb{C}}$ such that $\tau^{*} \bar{\zeta}$ is holomorphically isomorphic to $\zeta$, but there is no isomorphism $f_{0}$ from $\zeta$ to $\tau^{*} \bar{\zeta}$ that satisfies the condition

$$
\tau^{*} \overline{f_{0}} \circ f_{0}=\operatorname{Id}_{\zeta}
$$

see [4] for more details.

Let $g$ denote the genus of $X$. We recall below the definition of a real theta characteristic on $X$; see [4, p. 167].

DEFINITION 2.1. A point of $\operatorname{Pic}^{g-1}(X)_{\mathbb{R}}$ is called a real theta characteristic of $X$ if the corresponding line bundle $L$ over $X_{\mathbb{C}}$ satisfies the condition that $L^{\otimes 2} \cong K_{X_{\mathbb{C}}}$, where $K_{X_{\mathbb{C}}}$ is the cotangent line bundle of the complex curve $X_{\mathbb{C}}$. The set of all real theta characteristics on $X$ will be denoted by $\mathcal{S}(X)$. 
REMARK 2.2. In the above definition we do not demand that a real theta characteristic of $X$ is a real algebraic line bundle on $X$. Therefore the above definition of a real theta characteristic differs from the one given in [7], while it coincides with the definition in [4].

Let $\operatorname{Pic}^{0}\left(X_{\mathbb{C}}\right)_{2} \subset \operatorname{Pic}^{0}\left(X_{\mathbb{C}}\right)$ be the subgroup defined by all line bundles of order 2 . Define

$$
\mathcal{A}:=\operatorname{Pic}^{0}(X)_{\mathbb{R}} \cap \operatorname{Pic}^{0}\left(X_{\mathbb{C}}\right)_{2}
$$

to be the intersection.

We know that $\mathcal{S}(X)$ is nonempty [2, p. 61], [4, p. 164, Corollary 4.3]. The set $\mathcal{S}(X)$ is evidently a principal homogeneous space (also called a torsor) for $\mathcal{A}$ defined in (2.2). In other words, the group $\mathcal{A}$ acts freely transitively on the set $\mathcal{S}(X)$. The action of any $\xi \in \mathcal{A}$ on $\mathcal{S}(X)$ is defined by $L \longmapsto L \otimes \xi$.

The group $\mathrm{Pic}^{0}\left(X_{\mathbb{C}}\right)_{2}$ in (2.2) is canonically identified with $H^{1}\left(X_{\mathbb{C}}, \mathbb{Z} / 2 \mathbb{Z}\right)$. To see this, first note that

$$
H^{1}\left(X_{\mathbb{C}}, \mathbb{Z} / 2 \mathbb{Z}\right)=\operatorname{Hom}\left(\pi_{1}\left(X_{\mathbb{C}}\right), \mathbb{Z} / 2 \mathbb{Z}\right)
$$

A homomorphism $\left.\alpha: \pi_{1}\left(X_{\mathbb{C}}\right) \longrightarrow \mathbb{Z} / 2 \mathbb{Z}\right)$ gives a flat complex line bundle over $X_{\mathbb{C}}$ of order 2; in particular, $\alpha$ gives a holomorphic line bundle over $X_{\mathbb{C}}$ of order 2 . This identifies the group $\operatorname{Pic}^{0}\left(X_{\mathbb{C}}\right)_{2}$ with $H^{1}\left(X_{\mathbb{C}}, \mathbb{Z} / 2 \mathbb{Z}\right)$.

The involution $\tau$ in (2.1) gives an involution

$$
\widehat{\tau}: H^{1}\left(X_{\mathbb{C}}, \mathbb{Z} / 2 \mathbb{Z}\right) \longrightarrow H^{1}\left(X_{\mathbb{C}}, \mathbb{Z} / 2 \mathbb{Z}\right)
$$

defined by

$$
\alpha \longmapsto \tau^{*} \alpha
$$

The above identification of the group $\operatorname{Pic}^{0}\left(X_{\mathbb{C}}\right)_{2}$ with $H^{1}\left(X_{\mathbb{C}}, \mathbb{Z} / 2 \mathbb{Z}\right)$ sends the subgroup $\mathcal{A} \subset \mathrm{Pic}^{0}\left(X_{\mathbb{C}}\right)_{2}$ in (2.2) surjectively to the invariant subgroup

$$
H^{1}\left(X_{\mathbb{C}}, \mathbb{Z} / 2 \mathbb{Z}\right)^{\widehat{\tau}} \subset H^{1}\left(X_{\mathbb{C}}, \mathbb{Z} / 2 \mathbb{Z}\right)
$$

on which $\widehat{\tau}$ coincides with the identity map.

We noted earlier that $\mathcal{S}(X)$ is a principal homogeneous space for $\mathcal{A}$. Therefore, using the above identification $\mathcal{A}=H^{1}\left(X_{\mathbb{C}}, \mathbb{Z} / 2 \mathbb{Z}\right)^{\widehat{\tau}}$, we have an action of the group $H^{1}\left(X_{\mathbb{C}}, \mathbb{Z} / 2 \mathbb{Z}\right)^{\widehat{\tau}}$ on $\mathcal{S}(X)$,

$$
H^{1}\left(X_{\mathbb{C}}, \mathbb{Z} / 2 \mathbb{Z}\right)^{\widehat{\tau}} \times \mathcal{S}(X) \longrightarrow \mathcal{S}(X),
$$

which makes $\mathcal{S}(X)$ a principal homogeneous space for $H^{1}\left(X_{\mathbb{C}}, \mathbb{Z} / 2 \mathbb{Z}\right)^{\widehat{\tau}}$.

Given any automorphism $\sigma$ of the real curve $X$, let

$$
\widehat{\sigma}: X_{\mathbb{C}} \longrightarrow X_{\mathbb{C}}
$$

be the corresponding automorphism of $X_{\mathbb{C}}$. Since $\widehat{\sigma}$ is induced by an automorphism of $X$, it follows immediately that $\widehat{\sigma}$ commutes with the antiholomorphic involution $\tau$ in (2.1). This implies that for any $L \in \mathcal{S}(X) \subset \operatorname{Pic}^{g-1}\left(X_{\mathbb{C}}\right)$, the pullback $\widehat{\sigma}^{*} L$ is also an element of $\mathcal{S}(X)$. Therefore $\sigma$ acts on the set $\mathcal{S}(X)$ by sending any $L$ to $\widehat{\sigma}^{*} L$. 
Since $\tau$ and $\widehat{\sigma}$ commute, the subspace $H^{1}\left(X_{\mathbb{C}}, \mathbb{Z} / 2 \mathbb{Z}\right)^{\widehat{\tau}}$ in (2.4) is left invariant by the action of $\sigma$ on $H^{1}\left(X_{\mathbb{C}}, \mathbb{Z} / 2 \mathbb{Z}\right)$ that sends any $\beta \in H^{1}\left(X_{\mathbb{C}}, \mathbb{Z} / 2 \mathbb{Z}\right)$ to $\widehat{\sigma}^{*} \beta$. The map $\phi$ in (2.5) is clearly equivariant for the actions of $\sigma$ on $\mathcal{S}(X)$ and $H^{1}\left(X_{\mathbb{C}}, \mathbb{Z} / 2 \mathbb{Z}\right)^{\widehat{\tau}} \times \mathcal{S}(X)$; the action of $\sigma$ on $\mathcal{S}(X)$ is defined by $L \longmapsto \widehat{\sigma}^{*} L$, and $\sigma$ acts diagonally on $H^{1}\left(X_{\mathbb{C}}, \mathbb{Z} / 2 \mathbb{Z}\right)^{\widehat{\tau}} \times \mathcal{S}(X)$ through the above actions on $H^{1}\left(X_{\mathbb{C}}, \mathbb{Z} / 2 \mathbb{Z}\right)^{\widehat{\tau}}$ and $\mathcal{S}(X)$.

It is known that the hyperelliptic involution of a compact hyperelliptic Riemann surface $Z$ acts trivially on the set of all theta characteristics on $Z$; see [1, p. 288, 32(i)].

THEOREM 2.3. Let $X$ be a geometrically irreducible smooth projective curve, of genus at least 2 , defined over the field of real numbers, without any real points. Assume that $X$ admits a nontrivial automorphism $\sigma$. Then at least one of the following is true.

(1) The action of $\sigma$ on $\mathcal{S}(X)$ is nontrivial.

(2) The order of $\sigma$ is even, say $2 k$, and the involution $\tau \circ \widehat{\sigma}^{k}$ of $X_{\mathbb{C}}$ has a fixed point (see (2.6) for $\widehat{\sigma}$ ).

PRoOF. Let $g$ be the genus of $X$; so $g \geq 2$. Since $X$ does not have any real points, the involution $\tau$ in (2.1) does not have any fixed points. Therefore the quotient

$$
Y:=X_{\mathbb{C}} /\langle\tau\rangle
$$

is a smooth nonorientable surface.

Let $\sigma$ be a nontrivial automorphism of $X$ that acts trivially on $\mathcal{S}(X)$. As in (2.6), the corresponding automorphism of $X_{\mathbb{C}}:=X \times \mathbb{R} \mathbb{C}$ will be denoted by $\widehat{\sigma}$.

We noted above that the homomorphism $\phi$ in (2.5) is equivariant for the actions of $\sigma$. We also noted that $\mathcal{S}(X)$ is nonempty, and it is a principal homogeneous space for $H^{1}\left(X_{\mathbb{C}}, \mathbb{Z} / 2 \mathbb{Z}\right)^{\widehat{\tau}}$. Therefore the given condition that $\sigma$ acts trivially on $\mathcal{S}(X)$ implies immediately that the action of $\sigma$ on $H^{1}\left(X_{\mathbb{C}}, \mathbb{Z} / 2 \mathbb{Z}\right)^{\widehat{\tau}}$ defined by $\beta \longmapsto \widehat{\sigma}^{*} \beta$ is trivial.

Let

$$
H_{1}\left(X_{\mathbb{C}}, \mathbb{Z} / 2 \mathbb{Z}\right)^{\widehat{\tau}} \subset H_{1}\left(X_{\mathbb{C}}, \mathbb{Z} / 2 \mathbb{Z}\right)
$$

be the invariant part of the involution $\alpha \longmapsto \widehat{\tau}_{*}(\alpha)$ of $H_{1}\left(X_{\mathbb{C}}, \mathbb{Z} / 2 \mathbb{Z}\right)$. Similarly, $\sigma$ acts on $H_{1}\left(X_{\mathbb{C}}, \mathbb{Z} / 2 \mathbb{Z}\right)$ by sending any $\alpha$ to $\widehat{\sigma}_{*}(\alpha)$. Since $\sigma$ acts trivially on $H^{1}\left(X_{\mathbb{C}}, \mathbb{Z} / 2 \mathbb{Z}\right)^{\widehat{\tau}}$, by using the Poincaré duality pairing it follows immediately that $\sigma$ acts trivially on $H_{1}\left(X_{\mathbb{C}}, \mathbb{Z} / 2 \mathbb{Z}\right)^{\widehat{\tau}}$.

For any $\alpha, \beta \in H_{1}\left(X_{\mathbb{C}}, \mathbb{Z} / 2 \mathbb{Z}\right)$, their cap product, which is an element of $\mathbb{Z} / 2 \mathbb{Z}$, will be denoted by $\alpha \cdot \beta$. We recall that a linear basis

$$
\left\{\alpha_{1}, \beta_{1}, \ldots, \alpha_{g}, \beta_{g}\right\} \subset H_{1}\left(X_{\mathbb{C}}, \mathbb{Z} / 2 \mathbb{Z}\right)
$$

is called symplectic if $\alpha_{i} \cdot \beta_{j}=\delta_{i j}$ and $\alpha_{i} \cdot \alpha_{j}=0=\beta_{i} \cdot \beta_{j}$ for all $i, j$. The following lemma will be needed in the proof of the theorem.

LEMMA 2.4. There is a symplectic basis $\left\{\alpha_{1}, \beta_{1}, \ldots, \alpha_{g}, \beta_{g}\right\}$ of $H_{1}\left(X_{\mathbb{C}}, \mathbb{Z} / 2 \mathbb{Z}\right)$ such that $\widehat{\tau}_{*}\left(\beta_{i}\right)=\beta_{i}$ for all $1 \leq i \leq g$. 
PROOF. The real projective plane and the 2 -sphere will be denoted by $P$ and $S^{2}$ respectively. Let

$$
\pi^{\prime}: S^{2} \longrightarrow P
$$

be a universal covering map. The topological surface $Y$ in (2.7) is obtained from $P$ by removing the interiors of $g$ disjoint disks $D_{1}, \ldots, D_{g}$ and gluing in $g$ Möbius bands $M_{1}, \ldots, M_{g}$.

The orientable twofold cover of the Möbius band is an annulus. The surface $X_{\mathbb{C}}$ is obtained from the sphere $S^{2}$ by attaching $g$ disjoint 1-handles. Further, the boundary of each of the $g$ Möbius bands $M_{i} \subset Y$ has inverse image in $X_{\mathbb{C}}$, a pair of homologous curves. These two homologous curves are the boundary components of the corresponding annulus. We note that these two curves are also homologous to the central curve of the annulus. By the standard presentation for the homology of a surface, it follows that the central curves of the $g$ annuli form a symplectic half-basis (basis for a Lagrangian subspace) $\beta_{1}, \ldots, \beta_{g}$.

We have seen that, when $i \in[1, g]$, the elements $\beta_{i} \in H_{1}\left(X_{\mathbb{C}}, \mathbb{Z} / 2 \mathbb{Z}\right)$ are fixed by $\tau_{*}$. The lemma follows by completing the half-basis $\left\{\beta_{1}, \ldots, \beta_{g}\right\}$ to a symplectic basis.

Continuing with the proof of the theorem, we fix a symplectic basis

$$
\left\{\alpha_{1}, \beta_{1}, \ldots, \alpha_{g}, \beta_{g}\right\} \subset H_{1}\left(X_{\mathbb{C}}, \mathbb{Z} / 2 \mathbb{Z}\right)
$$

given by Lemma 2.4; so $\widehat{\tau}_{*}\left(\beta_{i}\right)=\beta_{i}$ for all $1 \leq i \leq g$. Since $\sigma$ acts trivially on $H_{1}\left(X_{\mathbb{C}}, \mathbb{Z} / 2 \mathbb{Z}\right)^{\widehat{\tau}}$, it follows that

$$
\widehat{\sigma}_{*}\left(\beta_{i}\right)=\beta_{i}
$$

for all $1 \leq i \leq g$.

Next, observe that for $1 \leq i \leq g$,

$$
\alpha_{i} \cdot \beta_{j}=\widehat{\sigma}_{*}\left(\alpha_{i}\right) \cdot \widehat{\sigma}_{*}\left(\beta_{j}\right)=\widehat{\sigma}_{*}\left(\alpha_{i}\right) \cdot \beta_{j}
$$

for all $j$. By elementary properties of the symplectic pairing it now follows that there are elements $a_{i j} \in \mathbb{Z} / 2 \mathbb{Z}$ such that

$$
\widehat{\sigma}_{*}\left(\alpha_{i}\right)=\alpha_{i}+\sum_{j=1}^{g} a_{i j} \beta_{j}
$$

for all $1 \leq i \leq g$.

Using (2.8), it follows by induction that

$$
\widehat{\sigma}_{*}^{n}\left(\alpha_{i}\right)=\alpha_{i}+\sum_{j=1}^{g} n a_{i j} \beta_{j}
$$

for all $n \in \mathbb{N}$.

As $\widehat{\sigma}$ is a holomorphic automorphism of $X_{\mathbb{C}}$, and $g \geq 2$, we know that $\widehat{\sigma}$ is periodic, that is, $\widehat{\sigma}^{n}=\operatorname{Id}_{X_{\mathbb{C}}}$ for some $n>1$.

First assume that the order $n$ of $\widehat{\sigma}$ is odd. From (2.9) we know that $a_{i j}=0$ for all $i, j \in[1, g]$. Thus, the automorphism $\widehat{\sigma}_{*}$ is the identity map of $H_{1}\left(X_{\mathbb{C}}, \mathbb{Z} / 2 \mathbb{Z}\right)$. 
This implies that $X_{\mathbb{C}}$ is a hyperelliptic Riemann surface, and $\widehat{\sigma}$ is the unique hyperelliptic involution of $X_{\mathbb{C}}$ (see [3, p. 495, Theorem 2.1]); recall that $\widehat{\sigma}$ is a nontrivial automorphism of $X_{\mathbb{C}}$. In particular, the order of $\widehat{\sigma}$ is 2 . But this contradicts the assumption that the order $n$ of $\widehat{\sigma}$ is odd.

Now consider the case where the order $n$ of $\widehat{\sigma}$ is even, say $n=2 k$. Assume that the second statement in the theorem is false. Therefore the involution $\tau \circ \widehat{\sigma}^{k}$ of $X_{\mathbb{C}}$ does not fix any point.

Since $\widehat{\sigma}$ commutes with $\tau$, we have an induced diffeomorphism $\bar{\sigma}$ of $Y=X_{\mathbb{C}} /\langle\tau\rangle$.

\section{LEMMA 2.5. The action of $\bar{\sigma}$ on $H_{1}(Y, \mathbb{Z} / 2 \mathbb{Z})$ is trivial.}

PROOF. We use the explicit description of the covering $X_{\mathbb{C}}$ of $Y$ given in the proof of Lemma 2.4. A basis for $H_{1}(Y, \mathbb{Z} / 2 \mathbb{Z})$ is given by the central circles of $P$ and the Möbius bands $M_{i}$ (see the proof of Lemma 2.4).

We first sketch a geometric proof. The inverse image of each of these central circles is a simple closed curve fixed (as a set) by $\tau$. It follows that $\tau$, hence $\widehat{\sigma}$, fixes the corresponding element in homology mod 2. One deduces that the homology classes of the central circles are fixed by $\bar{\sigma}$.

More formally, using Poincaré duality, the central circles $c_{i}$ of $P$ and $M_{i}$ represent elements of $H^{1}(Y, \mathbb{Z} / 2 \mathbb{Z})$. The pullback of these cohomology classes give elements of $H^{1}\left(X_{\mathbb{C}}, \mathbb{Z} / 2 \mathbb{Z}\right)$ represented (using Poincaré duality) by the inverse images $b_{i}$ of these curves. As these curves are fixed (as sets) by $\tau$, so are the elements in $H_{1}\left(X_{\mathbb{C}}, \mathbb{Z} / 2 \mathbb{Z}\right)$ they represent. It follows that $\widehat{\sigma}$ also fixes the corresponding elements in $H_{1}\left(X_{\mathbb{C}}, \mathbb{Z} / 2 \mathbb{Z}\right)$.

As $\widehat{\sigma}$ is a homeomorphism, it fixes the mod 2 fundamental class in $H_{2}\left(X_{\mathbb{C}}, \mathbb{Z} / 2 \mathbb{Z}\right)$. It follows that the elements in $H^{1}\left(X_{\mathbb{C}}, \mathbb{Z} / 2 \mathbb{Z}\right)$ represented by the curves $b_{i}$ are fixed by $\widehat{\sigma}$. We deduce that the elements of cohomology represented by the central circles of $P$ and the Möbius bands $M_{i}$ are fixed by $\bar{\sigma}$. By Poincaré duality, it follows that the action on $H_{1}(Y, \mathbb{Z} / 2 \mathbb{Z})$ is trivial. This completes the proof of Lemma 2.5.

Continuing with the proof of the theorem, consider the orbifold quotient $F$ of $Y$ by the involution $\bar{\sigma}^{k}$. Observe that $F$ is the quotient of $X_{\mathbb{C}}$ by the group generated by the involutions $\bar{\sigma}^{k}$ and $\tau$. As the orientation-reversing involutions in this group have no fixed points, the nonsmooth points of $F$ are all locally modelled on the quotient of the plane by a rotation of finite order (and not by a reflection).

It follows that the underlying surface of $F$ is nonorientable, since otherwise the orbifold cover $Y$ would be orientable. Hence $F$ is a connected sum of Möbius bands with isolated orbifold singularities and $Y$ is a twofold orbifold cover of $F$. Let $c_{i}$ denote the central circles of the Möbius band $Y$.

We claim that at least one such central curve $c_{i}$ lifts to $Y$. Otherwise, as $Y$ is a twofold cover and the curves $c_{i}$ generate $H_{1}(Y, \mathbb{Z} / 2 \mathbb{Z})$, we deduce that no orientationreversing curve lifts to $Y$, and hence $Y$ is orientable, a contradiction.

Suppose that the curve $c_{k}$ lifts to $Y$. Then its inverse image in $Y$ is a pair of disjoint simple curves $C_{k}$ and $C_{k}^{\prime}=\tau\left(C_{k}\right)$. We see that these represent different elements in 
$H_{1}(Y, \mathbb{Z} / 2 \mathbb{Z})$. Since $C_{k}$ is nonorientable, its mod 2 self-intersection number $C_{k} \cdot C_{k}$ is 1 mod 2. On the other hand, as $C_{k}$ and $C_{k}^{\prime}$ are disjoint, we have $C_{k} \cdot C_{k}^{\prime}=0$. Hence

$$
\left[C_{k}\right] \neq\left[C_{k}^{\prime}\right] \in H_{1}(Y, \mathbb{Z} / 2 \mathbb{Z}) .
$$

But by construction, $\tau$ maps $\left[C_{k}\right]$ to $\left[C_{k}^{\prime}\right]$. This contradicts Lemma 2.5. This completes the proof of the theorem.

\section{Examples}

In this section we will construct two examples. The first example shows that that there exist $X$ with a real point and admitting a nontrivial automorphism $\sigma$, such that:

(1) the action of $\sigma$ on $\mathcal{S}(X)$ is trivial; and

(2) $X /\langle\sigma\rangle \neq \mathbb{P}_{\mathbb{R}}^{1}$.

The second example shows that there exist $X$ with no real points and admitting a nontrivial automorphism $\sigma$, such that:

(1) the automorphism $\tau \circ \widehat{\sigma}^{k}$ has a fixed point;

(2) the action of $\sigma$ on $\mathcal{S}(X)$ is trivial; and

(3) $X /\langle\sigma\rangle \neq \mathbb{P}_{\mathbb{R}}^{1}$.

3.1. The first example. This will be obtained by constructing a compact connected Riemann surface $F$ with two commuting antiholomorphic involutions $a$ and $b$, so that $b$ acts trivially on $H_{1}(F, \mathbb{Z} / 2 \mathbb{Z})$. So $h:=a \circ b$ and $a$ have the same action in $H_{1}(F, \mathbb{Z} / 2 \mathbb{Z})$. In our example, $h$ will be a nontrivial automorphism, of the real algebraic curve $X$ defined by the pair $(F, a)$, satisfying the condition genus $(F /\langle h\rangle)>0$. More precisely, $h$ will be a nontrivial automorphism of $X$ that fixes all the real theta characteristics on $X$ pointwise. Since genus $(F /\langle h\rangle)>0$, the automorphism $h$ is not a hyperelliptic involution.

The compact Riemann surface $F$ will be constructed using hyperbolic geometry. Note that there is a bijective correspondence between hyperbolic surfaces and Riemann surfaces, with the hyperbolic isometries corresponding to the holomorphic and antiholomorphic self-maps.

Recall that there is a regular right-angled hexagon in the hyperbolic plane $\mathbb{H}$, which is unique up to an isometry of $\mathbb{H}$. Parameterize the sides of this hexagon by $\mathbb{Z} / 6 \mathbb{Z}=\{0,1,2,3,4,5\}$, preserving their circular orderings. Let $A_{1}, B_{1}, A_{2}$ and $B_{2}$ be four copies of this hexagon. For each $i \in\{1,2\}$, and

$$
n \in\{0,2,4\} \subset\{0,1,2,3,4,5\},
$$

identify the $n$th side of $A_{i}$ with the $n$th side of $B_{i}$ using the identity map. So, for $i \in\{1,2\}$, by these identifications we obtain a pair of pants $P_{i}$ with geodesic boundaries.

Next, for each $i=1,2$, identify the first side of $A_{i} \subset P_{i}$ with the third side of $A_{i} \subset P_{i}$ using the unique orientation-reversing isometry between the two sides. Similarly, identify the first side of $B_{i} \subset P_{i}$ with the third side of $B_{i} \subset P_{i}$ using the unique orientation-reversing isometry between the two sides. This gives a torus $T_{i}$ 
with one boundary component. The boundary of $T_{i}$ is the circle constructed from the fifth sides of $A_{i}$ and $B_{i}$.

Let $c_{i} \subset T_{i}$ be the geodesic circle which is the image of the union of the first sides in $A_{i}$ and $B_{i}$. Let $d_{i}$ be the simple closed curve in $T_{i}$ which is the image of the second side of $A_{i}$; note that in $P_{i}$, the second side of $A_{i}$ is identified with the second side of $B_{i}$. Finally, let $a_{i}\left(b_{i}\right)$ be the image in $T_{i}$ of the fifth side of $A_{i}\left(B_{i}\right)$. Therefore $a_{i}=A_{i} \cap \partial T_{i}$ and $b_{i}=B_{i} \cap \partial T_{i}$.

We now construct $F$ from $T_{1}$ and $T_{2}$ by identifying their boundary components in such a manner that the arcs $a_{1}$ and $a_{2}$ are identified, while $b_{1}$ is identified with $b_{2}$. More precisely, consider the unique orientation-reversing isometry from $a_{1}\left(b_{1}\right)$ to $a_{2}\left(b_{2}\right)$. Let $F$ be the quotient of the disjoint union of $T_{1}$ and $T_{2}$ constructed using these two isometries. Using the restriction of the hyperbolic metric on $\mathbb{H}$ to the regular hexagon, we obtain a hyperbolic metric on $F$. Therefore $F$ is equipped with a complex structure.

Consider the unique orientation-reversing isometries

$$
A_{1} \longrightarrow A_{2} \text { and } B_{1} \longrightarrow B_{2}
$$

that map $a_{1}$ to $a_{2}$ and $b_{1}$ to $b_{2}$, respectively. These two isometries and their inverses together induce an orientation-reversing isometry

$$
a: F \longrightarrow F
$$

of the quotient $F$ of the disjoint union of $A_{1}, A_{2}, B_{1}$ and $B_{2}$.

Similarly, we have an orientation-reversing isometry

$$
A_{1} \longrightarrow B_{1}
$$

$\left(A_{2} \longrightarrow B_{2}\right)$ that takes $a_{1}$ to $b_{1}\left(a_{2}\right.$ to $\left.b_{2}\right)$. These, together with their inverses, induce an orientation-reversing isometry

$$
b: F \longrightarrow F
$$

of the quotient $F$. Also define

$$
h:=a \circ b .
$$

Then $h$ is an orientation-preserving isometry of $F$. Hence, $h$ is a holomorphic automorphism of the Riemann surface $F$. We note that both $a$ and $b$ are involutions.

The isometry $b$ in (3.2) fixes the curves $c_{1}, c_{2}, d_{1}, d_{2}$ as sets, though not pointwise. As these generate $H_{1}(F, \mathbb{Z} / 2 \mathbb{Z})$, it follows that $b$ acts trivially on $H_{1}(F, \mathbb{Z} / 2 \mathbb{Z})$. Therefore the diffeomorphism $h$ in (3.3) fixes all the elements of $H_{1}(F, \mathbb{Z} / 2 \mathbb{Z})$ that are fixed by $a$. From this, it follows immediately that $h$ fixes all the elements of $H^{1}(F, \mathbb{Z} / 2 \mathbb{Z})$ that are fixed by $a$.

The pair $(F, a)$ defines a geometrically irreducible smooth projective curve of genus 2 defined over $\mathbb{R}$. Let $X$ denote the real curve defined by $(F, a)$. We note that the image in $F$ of the fifth side of $A_{1}$ is fixed pointwise by $a$. Therefore the real curve $X$ has real points. 
Clearly we have $a \circ b=b \circ a$, where $a$ and $b$ are constructed in (3.1) and (3.2), respectively. Therefore $h$ defines an automorphism of $X$. Let

$$
h^{\prime}: X \longrightarrow X
$$

be the automorphism of the real algebraic curve $X$ given by $h$. Since both $a$ and $b$ are involutions, it follows that $h^{\prime}$ is also an involution.

We will show that the above automorphism $h^{\prime}$ fixes all the real theta characteristics on $X$ pointwise.

We have already seen that $h$ fixes all the elements of $H^{1}(F, \mathbb{Z} / 2 \mathbb{Z})$ that are fixed by $a$. Since the homomorphism $\phi$ in (2.5) commutes with the action of $h^{\prime}$, to prove that $h^{\prime} \in \operatorname{Aut}(X)$ fixes all the real theta characteristics on $X$ pointwise, it suffices to show that there is one real theta characteristic on $X$ which is fixed by $h^{\prime}$.

To construct a real theta characteristic on $X$ which is fixed by $h^{\prime}$, let

$$
\left\{p_{i}^{0}, p_{i}^{1}\right\} \subset A_{i} \subset F
$$

be the two vertices of the first side of the hexagon $A_{i}$, where $i=1,2$. The midpoint of the second side of $A_{i}$ will be denoted by $q_{i}$. The two vertices of the fifth side of $A_{1} \subset F$ will be denoted by $x^{0}$ and $x^{1}$. Note the images in $F$ of the two vertices of the fifth side of $A_{2}, B_{1}$ and $B_{2}$ all coincide with the subset $\left\{x^{0}, x^{1}\right\} \subset F$.

LEMMA 3.1. Let $\mathcal{O}_{F}(D)$ be the holomorphic line bundle on $F$ given by the divisor

$$
D:=p_{1}^{0}+p_{1}^{1}+q_{1}-x^{0}-x^{1} .
$$

Then $\mathcal{O}_{F}(D)$ is a real theta characteristic on the real curve $X:=(F, a)$. The theta characteristic $\mathcal{O}_{F}(D)$ is fixed by the automorphism $h^{\prime}$ of $X$ defined in (3.4).

PROOF. We need an explicit construction of the hyperelliptic involution of $F$. For this, consider the reflections on the hexagons $A_{i}$ and $B_{i}$ about the geodesic line joining the midpoints of the second and fifth sides. These combine together to induce an orientation-reversing isometry

$$
c: F \longrightarrow F .
$$

Clearly, $c$ is an involution. Define

$$
f:=c \circ b,
$$

where $b$ is defined in (3.2). Therefore $f$ is an orientation-preserving isometry of $F$. Hence $f$ is a holomorphic automorphism of the Riemann surface $F$.

It is easy to see that the two automorphisms $c$ and $b$ commute. Since both $b$ and $c$ are involutions, it follows that $f$ is also an involution. It is easy to check that

$$
\operatorname{genus}(F /\langle f\rangle)=0 \text {. }
$$

Hence $f$ is the unique hyperelliptic involution of $F$.

The fixed points of the hyperelliptic involution of $F$ are precisely the Weierstrass points of $F$. Hence the fixed-point set

$$
F^{f}:=\left\{p_{1}^{0}, p_{1}^{1}, q_{1}, p_{2}^{0}, p_{2}^{1}, q_{2}\right\} \subset F
$$


is the set of Weierstrass points of $F$, where $p_{i}^{0}, p_{i}^{1}$ and $q_{i}$ are as in the statement of the lemma.

Also note that the subset $\left\{x^{0}, x^{1}\right\} \subset F$ is left invariant by the involution of $f$. As a consequence, the holomorphic line bundle on $F$ given by the divisor $x^{0}+x^{1}$ is isomorphic to the pullback $\widehat{f}^{*} \mathcal{O}_{\mathbb{P}_{\mathbb{C}}^{1}}(1)$, where

$$
\widehat{f}: F \longrightarrow F /\langle f\rangle \cong \mathbb{P}_{\mathbb{C}}^{1}
$$

is the quotient map, and $\mathcal{O}_{\mathbb{P}_{\mathbb{C}}^{1}}(1)$ is the unique line bundle of degree 1 over $\mathbb{P}_{\mathbb{C}}^{1}$.

Let $D^{\prime}:=p_{2}^{0}+p_{2}^{1}+q_{2}-x^{0}-x^{1}$ be the divisor on $F$. Let $\mathcal{O}_{F}\left(D^{\prime}\right)$ be the holomorphic line bundle over $F$ defined by $D^{\prime}$.

Using the above descriptions of $\widehat{f}^{*} \mathcal{O}_{\mathbb{P}_{\mathbb{C}}^{1}}(1)$ and the Weierstrass points of $F$, from the description, given in $[1$, p. 288, 32(i)], of the theta characteristics on $F$ it follows immediately that both $\mathcal{O}_{F}(D)$ and $\mathcal{O}_{F}\left(D^{\prime}\right)$ are theta characteristics on $F$; the line bundle $\mathcal{O}_{F}(D)$ is defined in the statement of the lemma. Furthermore, from [1, p. 288, 32(ii)] it follows immediately that $\mathcal{O}_{F}(D)$ and $\mathcal{O}_{F}\left(D^{\prime}\right)$ give the same theta characteristics on $F$; in other words, the holomorphic line bundle $\mathcal{O}_{F}\left(D^{\prime}\right)$ is isomorphic to $\mathcal{O}_{F}(D)$.

The map $a$ in (3.1) takes the divisor $D$ to the divisor $D^{\prime}$. Hence $\mathcal{O}_{F}(D)$ is a real theta characteristic on the real curve $X:=(F, a)$. The holomorphic isomorphism $h$ in (3.3) takes the divisor $D$ to the divisor $D^{\prime}$. Consequently, the isomorphism $h^{\prime}$ of $X$ constructed in (3.4) fixes the real theta characteristic defined by $\mathcal{O}_{F}(D)$. This completes the proof of the lemma.

As we noted prior to Lemma 3.1, it follows from Lemma 3.1 that $h^{\prime}$ fixes all the real theta characteristics on $X$ pointwise. On the other hand, we have

$$
\operatorname{genus}(F /\langle h\rangle)=1 \text {. }
$$

Hence $h^{\prime}$ is not the hyperelliptic involution of $X$. We also noted earlier that $X$ has real points. Therefore the automorphism $h^{\prime}$ of the real curve $X$ gives the first example.

It is easy to check that the holomorphic line bundle $\mathcal{O}_{F}(D)$ over $F$ in Lemma 3.1 defines a real algebraic line bundle over the real curve $X:=(F, a)$.

Taking the unique (up to isometry) regular right-angled $2(2 m+1)$-gon in the hyperbolic plane in place of the regular hexagon, higher-genus examples can be constructed similarly.

3.2. The second example. We note that the automorphism $c$ in (3.5) commutes with both $a$ and $b$ constructed in (3.1) and (3.2). Recall that $a$ commutes with $b$.

Consider the orientation-reversing involution

$$
\tau:=a \circ b \circ c
$$

of $F$. Let $X^{\prime}$ be the real curve defined by $(F, \tau)$. The involution $\tau$ does not have any fixed points. Hence $X^{\prime}$ does not have any real points. 
Now consider the automorphism $\sigma^{\prime}$ of $F$

$$
\sigma^{\prime}:=a \circ b \circ c \circ b=a \circ c
$$

which defines an involution of the real curve $X^{\prime}$. Note that $\tau \circ \sigma^{\prime}=b$ has fixed points.

We next note that both $b$ and $c$ fix all the Weierstrass points $F^{f}$ of $F$ pointwise (see (3.6)). Therefore Lemma 3.1 has the following corollary.

Corollary 3.2. Consider the divisor $D:=p_{1}^{0}+p_{1}^{1}+q_{1}-x^{0}-x^{1}$ in Lemma 3.1. The line bundle $\mathcal{O}_{F}(D)$ is a real theta characteristic on the real curve $X^{\prime}=(F, \tau)$, where $\tau$ is defined in (3.7). The theta characteristic $\mathcal{O}_{F}(D)$ is fixed by the automorphism of $X^{\prime}$ given by $\sigma^{\prime}$ constructed in (3.8).

Therefore $\sigma^{\prime}$ fixes all the real theta characteristics of $X^{\prime}$ pointwise. Hence $\left(X^{\prime}, \sigma^{\prime}\right)$ gives the second example.

\section{Odd theta characteristics on a Riemann surface}

We start with a topological lemma. Let $F$ be a compact connected Riemann surface with a holomorphic involution

$$
\gamma: F \longrightarrow F
$$

such that the quotient $F /\langle\gamma\rangle$ is the projective line $\mathbb{P}_{\mathbb{C}}^{1}$. Let

$$
f: F \longrightarrow \mathbb{P}_{\mathbb{C}}^{1}
$$

be the quotient map. Let $\mathcal{R} \subset \mathbb{P}_{\mathbb{C}}^{1}$ be the subset over which $f$ is ramified.

For any pair of ramification points $\{x, y\} \subset \mathcal{R}$, let $C(x, y)$ be a simple closed curve that separates this pair from the other ramification points. Then $f^{-1}(C(x, y))$ is a pair of simple closed curves. We shall see that these two curves represent the same element in $H_{1}(Y, \mathbb{Z} / 2 \mathbb{Z})$. Let $C^{\prime}(x, y)$ be one of these two curves.

LEMMA 4.1. The collection of curves $C^{\prime}(x, y)$, where $\{x, y\}$ run over all subsets of $\mathcal{R}$ of cardinality 2 , generate $H_{1}(F, \mathbb{Z} / 2 \mathbb{Z})$.

PROOF. Let $2 k$ be the cardinality of $\mathcal{R}$. We will prove the lemma by induction on $k$. For $k=0,1$, the surface $F$ is a sphere and hence $H_{1}(F, \mathbb{Z} / 2 \mathbb{Z})$ is trivial. Therefore the lemma is proved for $k=0,1$.

Now assume that the lemma holds for all $k \in\left[0, k_{0}\right]$, where $k_{0}$ is some positive integer. Take a pair $\left(F^{\prime}, f^{\prime}\right)$, where genus $\left(F^{\prime}\right)=k_{0}-1$ and

$$
f: F^{\prime} \longrightarrow \mathbb{P}_{\mathbb{C}}^{1}
$$

is a double cover. Pick a disk $D$ in $\mathbb{P}_{\mathbb{C}}^{1}$ disjoint from the $2 k_{0}$ ramification points of $f^{\prime}$. The inverse image of $D$ in $F^{\prime}$ is a pair of disks $D_{1}$ and $D_{2}$. As the ramified cover of a disk with two ramification points is an annulus, it follows that surface $F$, for $k=k_{0}+1$, is obtained from $F^{\prime}$ by removing the interiors of the disks $D_{1}$ and $D_{2}$ and attaching an annulus $A$. Furthermore, we can take $C(p, q)$ to be the boundary of the disk $D$. It follows that the inverse image of $C(p, q)$ consists of the boundary 
components of the annulus $A$, which are homologous curves in $H_{1}(F, \mathbb{Z} / 2 \mathbb{Z})$. Denote the class represented by these curves as $\beta$.

By the above, $F$ is obtained from $F^{\prime}$ by adding a 1-handle. Hence the rank of $H_{1}(F, \mathbb{Z} / 2 \mathbb{Z})$ is two more that that of $H_{1}\left(F^{\prime}, \mathbb{Z} / 2 \mathbb{Z}\right)$, with $\beta$ independent of $H_{1}\left(F^{\prime}, \mathbb{Z} / 2 \mathbb{Z}\right)$. Further, assume that $D$ is chosen in such a manner that the generators of $F^{\prime}$ given inductively are disjoint from $D_{1}$ and $D_{2}$. Then $\beta$ is disjoint from all the given generators of $H_{1}\left(F^{\prime}, \mathbb{Z} / 2 \mathbb{Z}\right)$. Hence if $\delta \in H_{1}\left(F^{\prime}, \mathbb{Z} / 2 \mathbb{Z}\right)$, then $\beta \cdot \delta=0$. (As in Section 2, for any $v, w \in H_{1}\left(F^{\prime}, \mathbb{Z} / 2 \mathbb{Z}\right.$ ), we will denote their cap product in $\mathbb{Z} / 2 \mathbb{Z}$ by $v \cdot w$.) Since the intersection pairing on the homology of a surface is a symplectic pairing, it follows that if $\alpha \in H_{1}(F, \mathbb{Z} / 2 \mathbb{Z})$ is an element with $\alpha \cdot \beta=1$, then the generators of $H_{1}\left(F^{\prime}, \mathbb{Z} / 2 \mathbb{Z}\right)$, together with $\alpha$ and $\beta$, generate $H_{1}(F, \mathbb{Z} / 2 \mathbb{Z})$.

Now let $r$ be a ramification point different from $p$ and $q$. We can choose $C(p, r)$ to intersect $C(p, q)$ transversally in two points $x$ and $y$. Observe that there is a curve consisting of arcs in $C(p, q)$ and $C(p, r)$ joining $x$ and $y$ that separates $p$ from the other ramification points, and hence does not lift to $F$. It follows that $C^{\prime}(p, r)$ and $C^{\prime}(p, q)$ intersect transversally in a single point. Hence, if $\alpha$ is the element represented by $C^{\prime}(p, r)$, then $\alpha \cdot \beta=1$.

It follows from the above that the generators of $H_{1}\left(F^{\prime}, \mathbb{Z} / 2 \mathbb{Z}\right)$ together with the elements $\alpha$ and $\beta$ represented by $C^{\prime}(p, r)$ and $C^{\prime}(p, q)$ respectively generate $H_{1}(F, \mathbb{Z} / 2 \mathbb{Z})$. The lemma follows by induction.

Let $Y$ be a compact connected Riemann surface of genus $g$. A holomorphic line bundle $L$ over $Y$ is called an odd theta characteristic if:

(1) $L^{\otimes 2}$ is holomorphically isomorphic to the holomorphic cotangent bundle $K_{Y}$;

(2) $\operatorname{dim} H^{0}(Y, L)$ is an odd integer.

The set of all odd theta characteristics on $Y$ will be denoted by $\mathcal{S}_{1}(Y)$. It is known that if $g>1$, then the cardinality of $\mathcal{S}_{1}(Y)$ is $2^{g-1}\left(2^{g}-1\right)$ [6, p. 190, Section 4].

Any holomorphic automorphism of $Y$ clearly acts on $\mathcal{S}_{1}(Y)$. Our aim in this section is to prove the following proposition.

PROPOSITION 4.2. Let $Y$ be a compact connected Riemann surface of genus at least 2 that admits a nontrivial holomorphic automorphism $\sigma$. If $\sigma$ fixes all the elements of $\mathcal{S}_{1}(Y)$ pointwise, then $Y$ is hyperelliptic and $\sigma$ is the unique hyperelliptic involution of $Y$.

PRoOF. Let $g$ be the genus of $Y$; so $g \geq 2$. Let $\mathcal{S}(Y)$ denote the set of all theta characteristics on $Y$. The set $\mathcal{S}(Y)$ is a principal homogeneous space for $H^{1}(Y, \mathbb{Z} / 2 \mathbb{Z})=H_{1}(Y, \mathbb{Z} / 2 \mathbb{Z})$ (the isomorphism is given by Poincaré duality). Let

$$
\mathcal{T}(Y) \subset H_{1}(Y, \mathbb{Z} / 2 \mathbb{Z})
$$

be the linear subspace generated by all elements $c \in H_{1}(Y, \mathbb{Z} / 2 \mathbb{Z})$ such that there exist

$$
\alpha, \beta \in \mathcal{S}_{1}(Y)
$$


with $\alpha=\beta+c$. It suffices to show that $\mathcal{T}(Y)=H_{1}(Y, \mathbb{Z} / 2 \mathbb{Z})$. Indeed, in that case the proposition follows from [3, p. 495, Theorem 2.1].

The parity of the theta characteristics remains fixed [2, p. 48, Theorem 1], [6, p. 184, Theorem] in a holomorphic family of compact connected Riemann surfaces with theta characteristics, parameterized by a connected space. Since the moduli space of Riemann surfaces of genus $g$ is connected, the integer $\operatorname{dim} \mathcal{T}(Y)$ depends only on $g$; in particular, $\operatorname{dim} \mathcal{T}(Y)$ is independent of the complex structure of $Y$. Therefore to prove that $\mathcal{T}(Y)=H_{1}(Y, \mathbb{Z} / 2 \mathbb{Z})$, we may assume that $Y$ is hyperelliptic.

Assume that $Y$ is a hyperelliptic Riemann surface. Let

$$
\gamma: Y \rightarrow Y
$$

be the hyperelliptic involution. Let

$$
f: Y \longrightarrow Y /\langle\gamma\rangle=\mathbb{P}_{\mathbb{C}}^{1}
$$

be the quotient map. So $f$ is ramified over $2 g+2$ points. Let $\mathcal{R} \subset \mathbb{P}_{\mathbb{C}}^{1}$ be the subset over which $f$ is ramified. For any pair of ramification points $\{x, y\} \subset \mathcal{R}$, let $C^{\prime}(x, y)$ be the closed curve in $Y$ considered in Lemma 4.1. Setting $m=0$ in [1, p. 288, 32(i)], we conclude from $\left[1\right.$, p. 288, 33] that the element in $H_{1}(Y, \mathbb{Z} / 2 \mathbb{Z})$ defined by $C^{\prime}(x, y)$ lies in $\mathcal{T}(Y)$ for all $\{x, y\} \subset \mathcal{R}$. Therefore the proposition follows from Lemma 4.1.

\section{Acknowledgement}

We thank S. Kallel for pointing out [5].

\section{References}

[1] E. Arbarello, M. Cornalba, P. A. Griffiths and J. Harris, Geometry of Algebraic Curves, Volume I, Grundlehren der Mathematischen Wissenschaften, 267 (Springer, New York, 1985).

[2] M. F. Atiyah, 'Riemann surfaces and spin structures', Ann. Sci. École Norm. Sup. 4 (1971), 47-62.

[3] I. Biswas, S. Gadgil and P. Sankaran, 'On theta characteristics of a compact Riemann surface', Bull. Sci. Math. 131 (2007), 493-499.

[4] B. H. Gross and J. Harris, 'Real algebraic curves', Ann. Sci. École. Norm. Sup. 14 (1981), 157-182.

[5] S. Kallel and D. Sjerve, 'Invariant spin structures on Riemann surfaces', http://arxiv.org/abs/math/ 0610568.

[6] D. Mumford, 'Theta characteristics of an algebraic curve', Ann. Sci. École. Norm. Sup. 4 (1971), 181-192.

[7] S. M. Natanzon, 'Spinors and differentials of real algebraic curves', in: Topology of Real Algebraic Varieties and Related Topics, American Mathematical Society Translations, Series 2, 173 (American Mathematical Society, Providence, RI, 1996), pp. 179-186.

\section{INDRANIL BISWAS, School of Mathematics, Tata Institute of Fundamental Research, Homi Bhabha Road, Mumbai 400005, India e-mail: indranil@math.tifr.res.in}

SIDDHARTHA GADGIL, Department of Mathematics, Indian Institute of Science, Bangalore 560003, India e-mail: gadgil@math.iisc.ernet.in 